\title{
Qualidade de vida em crianças obesas participantes de um programa de intervenção com exercícios físicos
}

\section{Quality of life in obese children participants on the physical exercise program intervention}

\author{
Raytta Silva Viana ${ }^{1}$ \\ Marina Melo Reis ${ }^{1}$ \\ Amanda Santos ${ }^{2}$ \\ Maria João da Cruz Lagoa ${ }^{3}$ \\ Alynne Christian Ribeiro Andaki ${ }^{1}$
}

\section{RESUMO}

A obesidade pediátrica pode afetar a qualidade de vida (QV) da criança em diversos aspectos da saúde, sejam eles físicos ou psicossociais e sua mensuração é considerada um importante parâmetro na avaliação e tratamento de crianças com excesso de peso. 0 objetivo do estudo foi avaliar o efeito de um programa de intervenção com exercício físico sobre a QV de crianças com sobrepeso ou obesidade. Estudo se caracterizou como um ensaio clínico não randomizado e controlado e a amostra foi composta por 45 crianças entre oito e 11 anos de idade, com Índice de Massa Corporal (IMC) acima do percentil 85 para idade e sexo. Os participantes foram divididos em 3 grupos, grupo controle $(n=22)$, grupo intervenção quadra $(n=11)$ e grupo intervenção piscina $(n=12)$. As crianças realizaram avaliação antropométrica para cálculo do IMC. A QV foi avaliada pelo questionário PedsQL versão genérica para crianças e pais, validado para a população brasileira. 0 teste ANCOVA univariada foi aplicado na comparação entre os grupos, com nível de significância $\alpha=5 \%$.Observou-se diferença significativa no autorrelato das crianças, entre os grupos, em relação ao domínio Atividade Escolar ( $\Delta \mathrm{GIP} \neq \mathrm{GC}, \Delta \mathrm{GIP}=\mathrm{GIQ} ; \mathrm{p}=0,05$ ) e verificou-se diferença significativa entre o autorrelato das crianças comparado aos questionários respondidos pelos pais, no domínio Atividade Escolar $(p=0,03)$ e $Q V$ geral $(p=0,02)$. Conclui-se que um programa de intervenção com exercícios físicos provocou efeito nos escores do domínio Atividade Escolar e QV geral de crianças com excesso de peso, com alterações significativas na percepção dos pais em relação as crianças.

\section{PALAVRAS-CHAVE}

Obesidade pediátrica, Qualidade de Vida, Exercício Físico.

\footnotetext{
${ }^{1}$ Universidade Federal do Triângulo Mineiro.

${ }^{2}$ Universidade Estadual de Maringá.

${ }^{3}$ Instituto Universitário de Maia.
} 


\section{ABSTRACT}

Pediatric Obesity affects the family and children quality of life (QoL) in several health aspects, physical or psychosocial, and it is measurement is considered an important parameter in the evaluation and treatment of overweight/obesity children. The aim of this study was to evaluate the effect of the physical exercise program on the QoL of the overweight or obesity children. Study was characterized as a non-randomized controlled clinical trial and the sample consisted of 45 8-11-year-oldchildren, with Body Mass Index (BMI) above 85 ${ }^{\text {th }}$ percentile for age and sex. The participants were divided into three groups, control group $(n=22)$, sport court intervention group $(n=11)$ and pool intervention group $(n=12)$. Children were evaluated in anthropometric parameters and the BMI was calculated. The QoL was evaluated by the PedsQL questionnaire version for children and parents, validated for the Brazilian population. The univariate ANCOVA test was applied in the comparison among groups, with significant level $\alpha=5 \%$. Observed significant different on the children self-report, among groups, regarding the school activity domain ( $\Delta$ GIP ₹ GC, $\Delta \mathrm{GIP}=\mathrm{GIQ} ; \mathrm{p}=0,05$ ) and found significant difference among children self-report compared the scores reported by parents on the school activity domain $(p=0,03)$ and general QoL $(p=0,02)$. Conclude that physical activity intervention program caused effects on scores of the school activity domain and general QoL in the children with excess weight, with significant changes on the perception of the parents in relationship by children.

\section{KEYWORDS}

Pediatric Obesity; Quality of Life; Physical Exercise. 


\section{INTRODUÇÃO}

A prevalência da obesidade infantil tem crescido de forma significativa em todo mundo, sendo considerada um grave problema de saúde pública (WHO, 2018).A obesidade na infância pode trazer consequências graves a saúde física devido a sua associação com diversas condições de morbidade (YUAN et al., 2020),além de graves consequências psicossociais como crises de ansiedade, transtornos de conduta, depressão, redução da autoestima, o que pode acarretar em prejuízo ao desenvolvimento da criança, refletindo, consequentemente em sua qualidade de vida (QV) (LEE et al., 2018; POETA, DUARTE e GIULIANO, 2010).

0 conceito de ?qualidade de vida? relativo à infância tem sido considerado subjetivo e multidimensional, considera-se várias dimensões psicossociais da criança e de sua família e a capacidade de desempenhar suas funções (BRASIL, 2003).A qualidade de vida relacionada ao estado de saúde é um conceito multifatorial que incorpora aspectos físicos, psicossociais e capacidade funcional da saúde ou da doença sobre a criança e sua família(VARNI, SEID, KURTIN, 2001; RHODES, et al., 2012).

O impacto negativo da obesidade na QV tem sido demostrado em diversos estudos, a maioria sugere que crianças com excesso de peso apresentam menor QV comparativamente à criança com peso saudável (SCHWIMMER, 2003; SWALLEN, REITHER, HAAS, 2005) além disso, a influência negativa do excesso de peso sobre o estado de saúde pode ter uma associação inversa entre obesidade e QV pediátrica (LEE et al., 2018; WILLIAMS, 2005). Porém, nem todos os aspectos que, especificamente, estão relacionados à obesidade estão projetados sobre a QV, contudo ela explora a percepção da saúde e bem-estar do indivíduo, pois apresenta domínios importantes de saúde como as funções físicas e psicossociais dificilmente encontrados por medidas fisiológicas e clínicas comuns (VARNI, SEID, KURTIN, 2001; KUNKEL, OLIVEIRA, PERES, 2009; TESTA, SIMONSON, 1996).

Estão evidenciados na literatura os benefícios promovidos pela prática regular de exercícios físicos sobre a saúde global do individuo, como a melhoria da aptidão cardiorrespiratória e muscular (GARCÍAHERMONOSO et al., 2019), saúde cardiometabólica e óssea, composição corporal (CARDEL et al., 2020) e alteração na QV de crianças (REIS et al., 2020). Assim, programas de intervenções com exercício físico exercem um importante papel na prevenção e no tratamento da obesidade (CARDEL et al., 2020) e por consequência influência a QV (KELLEY e KELLEY, 2013; REIS et al., 2020).

Considera-se a mensuração da QV um importante parâmetro na avaliação do tratamento de crianças com excesso de peso (LEE et al., 2018; POETA, DUARTE e GIULIANO, 2010) e para melhor compreensão da atuação do exercício físico sobre os aspectos biopsicossocias da QV na obesidade pediátrica, o presente estudo propõe avaliar o efeito de um programa de intervenção com exercício físico sobre a QV de crianças com sobrepeso ou obesidade.

\section{MÉTODOS}

O estudo caracterizou-se como um ensaio clínico não randomizado e controlado. Participaram do estudo crianças com idade entre oito e 11 anos, com Índice de Massa Corporal (IMC) acima do percentil 85 para idade e sexo, de acordo com os critérios da OMS (2007) ${ }^{22}$ para classificação de sobrepeso ou obesidade.A Escola Municipal Professor José Geraldo Guimarães foi selecionada por conveniência devido a ampla estrutura, na qual possui uma piscina semiolímpica $(25 \times 20 \mathrm{~m})$ com material de apoio necessário a intervenção (pranchas, água tubo, raias e halteres aquáticos), assim como um ginásio (área de $1.935 \mathrm{~m} 2$ ) e equipamentos esportivos e recreativos (bolas, cones, arcos).

Após aprovação do Comitê de Ética e Pesquisa com Seres Humanos da Universidade Federal do Triângulo Mineiro (CAAE: 63985317.9.0000.5154) e autorização da Secretaria Municipal de Educação de Uberaba, Minas Gerais, a avaliação antropométrica de todos os alunos matriculados na escola foi realizada. Assim, aqueles identificados acima do percentil 85, de acordo com os critérios da Organização Mundial de Saúde (ONNIS et al., 2007) foram convidados a conhecer a pesquisa (n=160). Após reunião com os pais, 49 responsáveis concordaram em participar do estudo e assinaram o Termo de Consentimento Livre e Esclarecido (TCLE). Posteriormente, as crianças foram aleatoriamente alocadas em um grupo controle (GC) n=17, grupo 
intervenção com atividade na quadra (GIQ) n=16, grupo intervenção com atividade na piscina (GIP) n=16. Após a mudança de rotina ocasionada nas duas primeiras sessões (horário de saída da escola alterado), houve solicitação de alguns pais para que os filhos fossem remanejados para o $\mathrm{GC}(\mathrm{n}=5)$ e não participassem da intervenção. O GC foi composto por $n=23$ crianças, enquanto o GIQ com n=13 e GIP ficou com n=13 no final da primeira semana de intervenção. Houve um total de 4 perdas no decorrer da intervenção e a amostra finalizou com $n=45$ participantes.

Os critérios de inclusão foram crianças com sobrepeso ou obesidade, com idade entre oito e 11 anos, que não estavam em dieta de restrição calórica, nem uso contínuo de medicamentos. Os critérios de exclusão foram iniciar a participação em outro programa de intervenção para perda de peso, não terem preenchido o mínimo de 50\% do PedsQL, não alcançar uma taxa de participação de $75 \%$ ou apresentar alguma deficiência física ou mental que impossibilitasse a participação. Os pais ou responsáveis foram alertados sobre a importância de as crianças manterem suas atividades habituais durante o período do experimento.

Os participantes foram submetidos a avaliações antropométricas e de QV nas semanas que antecederam o início da intervenção e nas semanas seguintes após a conclusão do período do programa (maio a novembro de 2017).A massa corporal (MC) foi obtida por balança eletrônica digital (Wiso, modelo W-721, Brasil) com capacidade máxima de $180 \mathrm{~kg}$ e precisão de $100 \mathrm{~g}$, conforme técnicas da Vigilância Alimentar e Nutricional (2004). A estatura foi obtida por meio de estadiômetro portátil (Sanny, PersonalCaprice, Sanny, Brasil) com comprimento de 2 metros e escala de $0,1 \mathrm{~cm}$, seguindo normas padronizadas. O IMC foi calculado com as medidas demassacorporal(MC) eestatura.Aclassificação das crianças com sobrepeso e obesidade foi realizada a partir do IMC/idade e sexo segundo critério da Organização Mundial da Saúde (ONNIS et al., 2007).

Foi utilizado o questionário pediátrico de Qualidade de Vida ? Pediatric Quality of Life Inventory (PedsQL 4.0) - versão genérica para crianças (VARNI, SEID e KURTIN, 2001), validado para a população brasileira. 0 instrumento possui dois questionários, um para a criança e outro para os pais ou responsáveis, neste estudo, foram consideradas as duas versões.0 instrumento inclui 23 tópicos segmentados em quatro domínios (físico, emocional, social e escolar), sendo respondidos seguindo cinco níveis de respostas $(0=$ nunca; 1 = quase nunca; 2 = algumas vezes; 3 = frequentemente; 4 = quase sempre), levando-se em consideração o último mês vivenciado pela criança. Cada item de respostas possui uma pontuação, sendo nunca $=100 ;$ quase nunca $=75$; algumas vezes $=25 \mathrm{e}$ quase sempre $=0$, o escore final do questionário é apresentado pela somatória de todos os itens seguido do cálculo da média dos domínios. Quanto mais elevado o escore do questionário, maior é considerada a qualidade de vida (KLATCHOIAN et al., 2008).

O programa de intervenção consistiu de exercícios físicos com atividades aeróbias, com características recreativa/esportiva, extra classe, durante 21 semanas. As atividades tiveram frequência de três vezes por semana, com duração de 60 minutos cada, totalizando 54 sessões, com a supervisão e acompanhamento de professores de Educação Física. Cada sessão consistia de alongamento/aquecimento (5 - 10 minutos), parte principal ? atividades físicas aeróbias, circuito de estações, jogos recreativos (30 - 40 minutos) e volta à calma (5 - 10 minutos). Os exercícios eram previamente planejados e desenvolvidos por um profissional de educação física e quatro graduandos do mesmo curso.

Os exercícios foram realizados buscando alcançar intensidade moderada a vigorosa e foi acompanhada individualmente, por meio da Escala de Borg Modificada para crianças (WATTERS et al., 2000) com o intuito de verificar o esforço percebido durante a sessão de treinamento. A escala de Borg Modificada é uma escala visual dividida em categorias, a tabela associa a uma ilustração um descritor textual do grau de dispnéia, à medida que essa sensação é aumentada a imagem corresponde a descrição da ilustração pelo indivíduo Para a manutenção das atividades na intensidade planejada, as crianças que relatassem pontuação inferior a 5 (muito leve ou leve) eram encorajadas e recebiam comandos dos professores para que aumentassem seu esforço.

Foi realizado o cálculo do delta de todas as variáveis (diferença entre valores pós intervenção e pré intervenção). A análise de variância univariada ANCOVA, com ajuste para a idade e pós-hoc de Bonferroni foi utilizada para verificar diferenças significativas entre 
grupos. As análises estatísticas foram realizadas com os dados previamente tabulados no Excel (Windows 2007) e testadas pelo software Statistical Package for Social Sciences SPSS 24.0, a um nível de significância $\alpha=5 \%$.

\section{RESULTADOS}

Participaram do estudo $n=45$ crianças, $55,1 \%$ do sexo feminino. A média de idade dos grupos foram GIQ $9,0 \pm 0,5$, GIP $9,3 \pm 1,0$ e GC $9,1 \pm 1,2$ anos.

Na Tabela 1 foram apresentados os deltas dos dados antropométricos por grupos. Embora o GIQ apresentasse valores de delta superiores para a estatura, massa corporal e IMC em relação aos grupos GIP e GC, não houve diferença significativa entre os grupos $(p>0,05)$.

Tabela 1. Delta das características antropométricas de crianças com sobrepeso e obesidade dos grupos intervenção quadra, piscina e controle.

\begin{tabular}{lllllllllll}
\hline Variáveis & \multicolumn{3}{c}{ GIQ } & \multicolumn{3}{c}{ GIP } & \multicolumn{3}{c}{ GC } \\
\hline & $\mathrm{n}$ & $\Delta$ & $\mathrm{Dp}$ & $\mathrm{n}$ & $\Delta$ & $\mathrm{Dp}$ & $\mathrm{n}$ & $\Delta$ & $\mathrm{Dp}$ & $p$-valor \\
\hline Estatura (cm) & 11 & 3,42 & 1,42 & 12 & 2,34 & 1,28 & 22 & 2,76 & 1,91 & 0,32 \\
Massa Corporal (Kg) & 11 & 6,60 & 5,35 & 12 & 6,13 & 3,50 & 21 & 5,27 & 3,66 & 0,67 \\
IMC (Kg/m2) & 11 & 0,53 & 4,56 & 12 & 2,27 & 2,99 & 21 & 0,68 & 4,99 & 0,58 \\
\hline
\end{tabular}

p-valor obtido pelo teste de ANCOVA Univariada.

Legenda: GIQ= Grupo Intervenção Quadra; GIP = Grupo Intervenção Piscina; GC = Grupo Controle; IMC = Índice de Massa Corporal; $\mathrm{n}=$ Número de Amostra; $\Delta$ = Delta (valores pós experimento - valores pré experimento); $\mathrm{Dp}$ = Desvio Padrão

Comparativo entre os deltas $(\Delta)$ dos escores dos domínios da $\mathrm{QV}$ entre os grupos e entre pais e filhos foram apresentadosna Tabela 2. Foiobservado diferença significativa no autorrelato das crianças,entre os grupos,em relação ao domínio Atividade Escolar ( $\Delta$ GIP ₹ GC, $\Delta \mathrm{GIP}=\mathrm{GIQ} ; \mathrm{p}=0,05$ ), não houve diferença entre os grupos para o relato dos pais $(p>0,05)$. Verificou-se diferença significativa entre o autorrelato das crianças comparado aos questionários respondidos pelos pais, quanto ao domínio Atividade Escolar $(\mathrm{p}=0,03)$ e QV geral $(p=0,02)$, independente do grupo,houve superioridade nos valores de delta apresentado pelos pais em relação aos filhos. Os deltas dos domínios de QV entre os GIP vs GIQ vs GC, relatado pelos pais e crianças não apresentaram diferença significativa. Não houve interação entre os valores de delta entre pais, filhos e grupos avaliados $(p>0,05)$, Tabela 2 .

\section{DISCUSSÃo}

Nossosprincipais achadosrevelaram que houve diferença significativa entre os escores do domínio Atividade Escolar e QV Geral do relato das crianças, comparado ao dos pais. Os valores de delta da Atividade Escolar e QV geral identificados pelos pais foram todos positivos e superestimaram a QV relatada pelos filhos. Assim, a intervenção com exercícios aeróbios provocou efeito na QVde crianças obesas na percepção dos pais. Houve também diferença significativa entre os grupos no autorrelato das crianças para o domínio Atividade Escolar.

O domínio Atividade Escolar e QVapresentaram diferença significativa entre pais e filhos, ou seja, maiores escores no aspecto da atividade escolar e QV foram encontrados no momento pre intervenção quando comparado ao momento pós intervenção (delta negativo)autorrelato das crianças, e avaliação contrária foi descrita pelos pais (delta positivo). Pesquisas sugerem que talvez esta questão se deva ao fato dos pais apresentarem maior nível de interesse, cuidado e preocupação com os hábitos e desempenho das crianças antes do início de um tratamento pediátrico (WILLIAMS, 2005; KUNKEL, OLIVEIRA, PERES, 2009).Este domínio apresenta questões como "É difícil prestar atenção à aula", "Eu esqueço coisas", "Tenho dificuldade para acompanhar minha turma em tarefas escolares", todas relacionadas com deficit de atenção. 


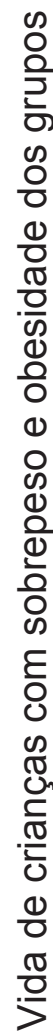

198

$\frac{\frac{1}{0}}{\frac{0}{0}}$

ণ

을

응

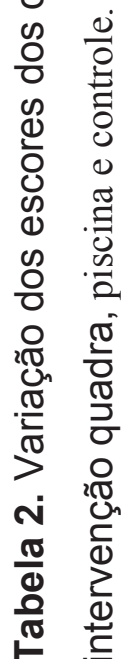

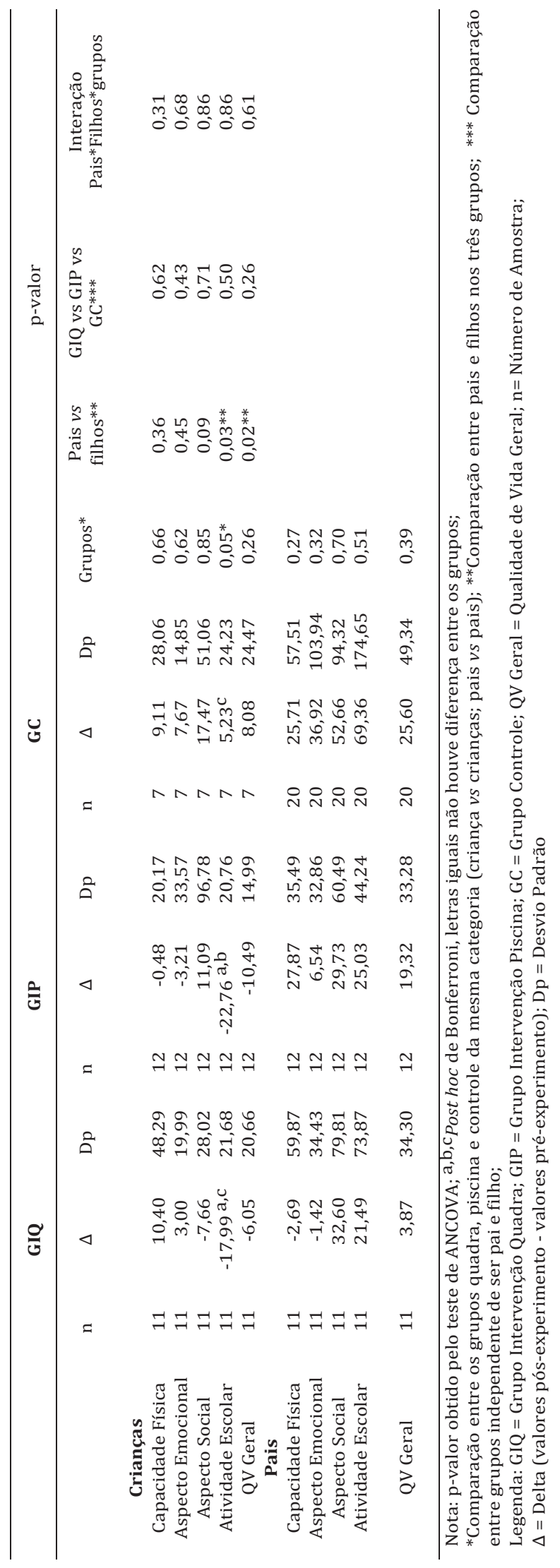


Estudo com crianças brasileiras, na faixa etária de sete a 11 anos de idade, comprovou que o exercício pode ajudar a melhorar a concentração e a função cognitiva (MELO, 2017). Além disso, o instrumento traz mais duas questões no domínio atividade escolar relacionada à saúde física ? Eu falto à aula por não estar me sentindo bem? ? Eu falto à aula para ir ao médico ou hospital?

Os dados encontrados são relevantes, uma vez que a perspectiva dos pais sobre a saúde dos filhos é tão importante quanto à percepção da própria criança sobre seu estado (JANICKE, FINNEY, RILEY, 2001). A percepção dos pais pode impulsionar o processo de tomada de decisão que implica na busca de cuidados de saúde em favor das crianças (ZELLER, MODI, 2006). Segundo Williams (2005) qualquer resultado positivo ainda que pequeno, sobre a QV da criança obesa é significativo, é essencial que não somente as crianças, mas que os pais percebam as implicações que o excesso de peso pode causar na saúde para procurarem meios que auxiliem na reduçãode massa gorda.

Nossos resultados apresentam similaridade com outros estudos realizados com o mesmo instrumento, nos quais a percepção dos jovens sobre $\mathrm{QV}$ foi diferente da apontada pelospais (KUNKEL, OLIVEIRA, PERES, 2009; SCHWIMMER, 2003; BASS, BERESIN, 2009).Para crianças obesas a QV pode ser influenciada por situações de sua rotina, trazendo impactos sobre as pessoas próximas e a si (WALLANDER, SCHMITT, KOOT, 2001). Dessa maneira, a QV geral ainda que percebida pelos pais sobre seus filhos, têm real importância para verificar a QV na obesidade pediátrica. A criança necessita ter uma compreensão global das perguntas em instrumentos que avaliam a QV, para conseguir formular uma resposta. Além disso, dificuldades em responder perguntas sobre um período de tempo específico são frequentes entre as crianças impossibilitando uma informação mais precisa sobre a QV. Ainda, os pais têm demonstrado melhor capacidade de percepção em alguns domínios da QV relatada sobre os filhos, do que as próprias crianças. Sendo assim, para que a resposta seja considerada mais acurada, grande parte dos instrumentos para avaliação da QV em crianças é respondido pelos pais ou responsáveis (SOARES, et al., 2003).

Entre os grupos, para o autorrelato das crianças, o aspecto escolar apresentou diferença significativa. 0
GIP e GIQapresentaresm delta negativos enquanto que GC apresentou valores de delta positivo, contrariando nossa hipótese inicial.Não houve diferença estatisticamente significativa entre os grupos intervenção (Piscina e Quadra), assim como não foi demonstrada diferença entre os grupos GIQ e GC. Além do exposto, de acordo com o estudo de MELO (2017) a atividade escolar em comparação aos outros domínios da QV não é prejudicada em crianças com obesidade. Assim, acreditamos que os escores da atividade escolar poderiam ser aumentados e isto proporcionaria uma elevação da QV geral, fato que é de grande importância para obesidade pediátrica, uma vez que a QV é encontrada em escores mais baixos nesta população, ainda que este aspecto seja menos afetado.

A influência do excesso de peso sobre a QV das crianças é de relevante diante da proporção alarmante que a obesidade pediátrica tem alcançado (ONIS, et al., 2010). Sugere-se que mais estudos experimentais sejam realizados com a população em questão e suas famílias, levando em consideração os aspectos da vida que acarretam maiores prejuízos a QV das crianças com obesidade.

O questionário PedsQL foi criado em módulos para verificar a $Q V$ relacionada à saúde de crianças de maneira genérica e para comorbidades específicas. Contudo, o questionário tem sido amplamente utilizado para população infantil e com distintas doenças. Porém, durante a aplicação do PedsQLverificamos anecessidade de um questionário específico à obesidade pediátrica, com questionamentos voltados ao excesso de peso, uma vez que a obesidade é uma doença multifatorial. Dessa forma, faz-se importante a ampliação, desenvolvimento e aplicação de instrumentos de avaliação da qualidade de vida associada à obesidade, com intuito de que os resultados proporcionem melhores considerações às crianças e aos familiares e auxiliem os profissionais da saúde na prevenção e tratamento da obesidade infantil.

O presente estudo apresentou algumas limitações. A randomização inicial dos grupos não sebnmanteve, alguns pais solicitaram realocação das crianças do GE para o GC, pois osnmesmos não tinham disponibilidade para acompanhamento após horário regular das aulas. Outra limitação refere-se ao fato do experimento focar em diferentes exercícios físicos como meio do controle do excesso de peso sem acompanhamento de outros 
profissionais da área da saúde, como por exemplo uma nutricionista. 0 tempo e o tipo de intervenção ofertada podem ter sido insuficientes para modificação nos domínios de QV estudados. 0 número de retornos dos questionários respondidos foi abaixo do esperado, o que pode ter limitado resultados neste estudo.

Os achados do presente estudo apresentam pontos fortes como a oferta de 180 minutos semanais, de exercício físico de intensidade moderada a vigorosa, auxiliando essa população no aumento do nível de atividade física. Os exercícios foram eficientes na manutenção da motivação das crianças para permanência no programa, contribuindo para uma adesão de $95 \%$ dos participantes.

Conclui-se que um programa de intervenção com exercícios físicos provocou efeito nos escores do domínio Atividade Escolar e QV geralde crianças com excesso de peso, com alterações significativas na percepção dos paisem relação as crianças. 


\section{REFERÊNCIAS}

BALAGOPAL P.; DE FERRANTI S. D.; COOK S.; DANIELS S. R.; GIDDING S. S.; HAYMAN L. L.; MCCRINDLE B. W.; MIETUSSNYDER M. L.; STEINBERGER J. Nontraditional Risk Factors and Biomarkers for Cardiovascular Disease: Mechanistic, Research, and Clinical Considerations for Youth. American Heart Association - Circulation, v. 123, n. 23, p. 2749-69, jun. 2011.Disponível em: <https://www.ahajournals.org/ doi/10.1161/CIR.0b013e31821c7c64>.

BASS L. M.; BERESIN R. Quality of life in obese children. Einstein, São Paula, v. 7, n. 3, p. 295-301, 2009.

BRASIL T.; FERRIANI V.; MACHADO C. Inquérito sobre a qualidade de vida relacionada à saúde em crianças e adolescentes portadores de artrites idiopáticas juvenis. J Pediatr, v. 79, n. 1, p. 63-8, 2003.

BUTTITTA M.; ILIESCU C.; ROUSSEAU A.; GUERRIEN A. Quality of life in overweight and obese children and adolescents: a literature review. Qual Life Res, v. 23, n. 4, p. 1117-39, may. 2014.

AMBULI V. M.; MUSIU M. C.; INCANI M.; PADERI M.; SERPE R.; MARRAS V.; COSSU E.; CAVALHO M. G.; MARIOTTI S.; LOCHE S.; BARONI M. G. Assessment of Adiponectin and Leptin as Biomarkers of Positive Metabolic Outcomes after Lifestyle Intervention in Overweight and Obese Children. Journal Clinic Endocrinology Metabolism, v. 93, n. 8, p. 3051-7, aug. 2008. Disponível em: <https://academic.oup.com/jcem/articlelookup/doi/10.1210/jc.2008-0476>.

CARDEL, M. I. et al. Obesity Treatment Among Adolescents: A Review of Current Evidence and Future Directions. JAMA Pediatrics, v. 174, n. 6, p. 609, 1 jun. 2020.

DUNCAN M. J.; AL-NAKEEB Y.; NEVILL A. M. Effects of a 6-week circuit training intervention on body esteem and body mass index in British primary school children. Body Image, v. 6, n. 3, p. 216-20, jun. 2009.

FULLERTON G.; TYLER C.; JOHNSTON C. A.; VINCENT J. P.; HARRIS G. E.; FOREYT J. P. Quality of Life in Mexican-American Children Following a Weight Management Programan. Obesity, v. 15, n. 11, p. 2553-6, nov. 2007.

GARCÍA-HERMOSO, A. et al. Health-related physical fitness and weight status in 13- to 15-year-old Latino adolescents. A pooled analysis. Jornal de Pediatria, v. 95, n. 4, p. 435-442, jul. 2019.

JANICKE D. M.; FINNEY J. W.; RILEY A. W. Children's health care use: a prospective investigation of factors related to careseeking. Medicine Care, v. 39, n. 9, p. 990-1001, sep. 2001.

JUONALA M.; MAGNUSSEN C. G.; BERENSON G. S.; VENN A.; BURNS T. L.; SABIN M. A.; SRINIVASAN S. R.; DANIELS S. R.; DAVIS P. H.; CHEN W.; SUN C.; CHEUNG M. Childhood Adiposity,
Adult Adiposity, and Cardiovascular Risk Factors. New England Journalof Medicine, v.365, n. 20, p. 1876-85, nov. 2011. Disponível em: <http://www.nejm.org/doi/abs/10.1056/ NEJMoa1010112>.

KAHN B. B.; FLIER J. S. Obesity and insulin resistance. Journal Clinic Investigation, v. 106, n. 4, p. 473-81, aug. 2000. Disponível em: <http://www.jci.org/articles/view/10842>.

KELLEY G. A.; KELLEY K. S. Effects of Exercise in the Treatment of Overweight and Obese Children and Adolescents: A Systematic Review of Meta-Analyses. Journal Obesity, v. 2013, p. 1-10, 2013

KUNKEL N.; OLIVEIRA W. F. DE.; PERES M. A. Execesso de peso e qualidade de vida relaciona à saúde em adolescentes de Florianópolis, SC. Revista Saúde Pública, v.43, n. 2, p. 226-35, apr. 2009. Disponível em: <http://www.scielo.br/scielo. php?script=sci_arttext\&pid=S0034-89102009000200003\&ln $\mathrm{g}=\mathrm{pt} \& \operatorname{tnn} \mathrm{g}=\mathrm{pt}>$.

KLATCHOIAN D. A.; LEN C. A.; TERRERI M. T. R. A.; SILVA M.; ITAMOTO C.; CICONELLI R. M.; VARNI J. W.; HILÁRIO M. O. E. Qualidade de vida de crianças e adolescentes de São Paulo: confiabilidade e validade da versão brasileira do questionário genérico Pediatric Quality of Life InventoryTM versão 4.0. Journal Pediatric, v. 84, n. 4, p. 308-15, aug. 2008.

LEE C. T; LIN C. Y.; STRONG C.; LIN Y. F.; CHOU, Y. Y.; TSAI M. C.; Metabolic correlates of health-related quality of life among overweight and obese adolescents. BMC Pediatric, v. 18, n. 1, p. 25, dec. 2018.

MARTIN A.; SAUNDERS D. H.; SHENKIN S. D.; SPROULE J. Lifestyle intervention for improving school achievement in overweight or obese children and adolescents. Cochrane Database Systematic Review, v. 14, n. 3, mar, 2014.

MELO J. C. M. DE. Efeitos de um programa de intervenção sobre indicadores da aptidão física e atividade física habitual em meninos com excesso de peso e obesidade. Faculdade de Desporto da Universidade do Porto; 2017.

ONIS M.; ONYANGO A. W.; BORGHI, E.; SIYAM A.; NISHIDA C.; SIEKMANN J. Development of a WHO growth reference for school-aged children and adolescents. Bull World Health Organ. v. 85, n. 9, p.600-617, Sep. 2007."

POETA L. S.; DUART M. DE F. DA S.; GIULIANO I. DE C. B. Qualidade de vida relacionada à saúde de crianças obesas. Revista Associação Médica Brasileira, v. 56, n.2, p. 168-72, 2010.

RAVENS-SIEBERER U.; REDEGELD M.; BULLINGER M. Quality of life after in-patient rehabilitation in children with obesity. International Journal Obesity, v. 25, n. S1, p. 63-5, may. 2001. Disponível em: <http://www.nature.com/articles/0801702>. 
REIS, M. DE M. et al. Alterações na antropometria e na qualidade de vida relacionada à saúde após um programa de exercícios aquáticos em crianças com sobrepeso e obesidade. Revista Brasileira de Atividade Física \& Saúde, v. 24, p. 1-7, 31 maio 2020.

RHODES E. T.; GORAN M. I.; LIEU T. A.; LUSTIN R. H.; PROSSER L. A.; SONGER T. J.; WEIGENSBERG M. J.; WEINSTOCK R. S.; GONZALEZ T.; RAWLUK K.; ZOGHBI R. M.; LUDWIG D. S.; LAFFEL L. M. Health-Related Quality of life in adolescents with or at Risk for Type 2 Diabetes Mellitus. Journal Pediatric, v. 160, n. 6, p. 911-7, jun 2012. Disponível em: <https:// linkinghub.elsevier.com/retrieve/pii/S0022347611011607>.

SOARES D. B.; PORTO E.; MARCO A. DE; AZONI C. A. S.; CAPELATTO I. V. Influência da atividade física no desempenho motordecriançascom queixas dedificuldadesdeaprendizagem. Revista CEFAC, v. 17, n. 4, p. 1132-42, aug. 2015.

SCHWIMMER J. B. Health-Related Quality of Life of Severely Obese Children and Adolescents. JAMA, v. 289, n. 14, p. 1813, apri. 2003.

SWALLEN, K.C.; REITHER E. N.; HAAS S. A. M. A. Overweight, Obesity, and Health-Related Quality of Life Among Adolescents: The National Longitudinal Study of Adolescent Health. Pediatrics, v. 115, n. 2, p. 340-7, feb. 2005.

TESTA M. A.; SIMONSON D. C. Assessment of Quality-of-Life Outcomes. New England Journal of Medicine, v. 334, n. 13, p. 835-40, mar. 1996. Disponível em: <http://www.nejm.org/ doi/abs/10.1056/NEJM199603283341306>.

VALADÃO M. Os efeitos de um programa de atividade física nos marcadores inflamatórios e na densidade mineral óssea de crianças obesas. Faculdade de Desporto da Universidade do Porto, Universidade do Porto; 2015.
VANHELST J.; FARDY P. S.; BÉGHIN L.; BUI-XUAN G.; MIKULOVIC $J$. Strategies in intervention programmes for obese youth: implication of the age and the type of physical activities. Clinic Physiology Function Imaging, v. 35, n. 1, p. 17-20, jan. 2013. Disponível em: <http://doi.wiley.com/10.1111/cpf.12112>

VARNI J. W.; SEID M.; KURTIN P. S. PedsQL 4.0: reliability and validity of the Pediatric Quality of Life Inventory Version 4.0 generic core scales in healthy and patient populations. Medicine care, v. 39, n. 8, p. 800-12, aug. 2001. Disponível em:<http://www.ncbi.nlm.nih.gov/pubmed/11468499>.

WALLANDER J. L.; SCHMITT M.; KOOT H. M. Quality of life measurement in children and adolescents: issues, instruments, and applications. Journal Clinic Psychology, v. 57, n. 4, p. 571 85, apri. 2001

WATERS E.; DOYLE J.; WOLFE R.; WRIGHT M.; WAKE M.; SALMON L. Influence of Parental Gender and Self-Reported Health and Illness on Parent-Reported Child Health. Pediatrics, v. 106, n. 6, p. 1422-8, dec. 2000.

WILLIAMS J. Helath-Related Quality of Life of Overweight and Obese Children. JAMA, v. 293, n. 1, p. 70, jan. 2005. Disponíve e m: < http://jama.jamanetwork.com/article. aspx?doi=10.1001/jama.293.1.70>.

WHORLD HEALTH ORGANIZATION.Obesity andoverweight fact sheet.WHO; Geneva, 2018

YUAN, Y. et al. Body Mass Index Trajectories in Early Life Is Predictive of Cardiometabolic Risk. The Journal of Pediatrics, v. 219, p. 31-37.e6, abr. 2020

ZELLER M. H.; MODI A. C. Predictors of Health-Related Quality of Life in Obese Youth. Obesity, v. 14, n. 1, p. 122-30, jan. 2006 